\title{
Modificações sensoriais em abacaxi 'Pérola' armazenado à temperatura ambiente
}

\author{
Sensory modifications in pineapple stored at room temperature
}

\author{
Ana Carolina Almeida MIGUEL ${ }^{1 \star}$, Camila ABRAHÃO'1, João Ricardo Pecini Stein DIAS ${ }^{1}$, Marta Helena Fillet SPOTO ${ }^{1}$
}

\begin{abstract}
Resumo
O objetivo deste trabalho foi avaliar as alterações nos principais atributos sensoriais de abacaxis 'Pérola' em quatro estádios de maturação durante o armazenamento à temperatura ambiente. A cada dois dias avaliaram-se a aparência, o odor, o sabor e a textura de frutos de abacaxi 'Pérola' nos estádios de maturação, "verde", "pintado", "colorido" e "amarelo", adquiridos na CEAGESP (SP) e armazenados à temperatura ambiente $\left(23,4 \pm 1,7^{\circ} \mathrm{C} ; 70 \% \mathrm{UR}\right)$, por 6 dias. O estádio de maturação afetou a qualidade sensorial do abacaxi. A qualidade sensorial foi afetada negativamente pelo estádio de maturação, atingindo maior grau de qualidade nos pontos "pintado" e "colorido".

Palavras-chave: Ananas comosus; qualidade; pós-colheita; conservação.
\end{abstract}

\begin{abstract}
In the present work the sensory modifications in the pineapple's four ripeness stages during room temperature storage were evaluated. Every two days, appearance, smell, flavor, and texture of the pineapple in the ripeness stages, "green", "spotted", "colored" and "yellow" obtained from CEAGESP (São Paulo State, Brazil) and stored at room temperature $\left(23.4 \pm 1.7^{\circ} \mathrm{C} ; 70 \% \mathrm{RH}\right)$ were evaluated for 6 days. The ripeness stages influenced the pineapple sensory quality negatively reaching the highest values in the quality in the "spotted" and "colored" stages. Keywords: Ananas comosus; quality; postharvest; storage.
\end{abstract}

\section{Introdução}

A produção mundial de abacaxi foi de 16,3 milhões de toneladas em 2004 sendo que Tailândia, Filipinas, Costa Rica e Brasil figuram como os maiores produtores (AGRIANUAL, 2007).

Os abacaxis brasileiros são representados principalmente pelas cultivares Pérola e Smooth Cayenne, sendo que a primeira é considerada insuperável devido à sua polpa suculenta e saborosa, bem como menores teores de acidez e fibras, características que fazem com que os frutos apresentem grande potencial de comercialização internacional (SANTANA et al., 2004; SOUTO et al., 2004).

Segundo Reinhardt et al. (2000), na prática, o desenvolvimento do fruto e a sua aparência, sobretudo a coloração da casca, são os principais indicadores do ponto de colheita do abacaxi. Embora este método não seja efetivo, haja vista que a coloração da casca sofre interferência da temperatura (CHITARRA, M. I. F.; CHITARRA, A. B., 2005), ainda é considerado fator principal na avaliação do amadurecimento da fruta no campo (GIACOMINO, 2001).

Os atributos de qualidade dizem respeito à aparência, sabor e odor, textura, valor nutritivo e segurança dos produtos. Tais atributos têm importância variada, de acordo com os interesses de cada segmento da cadeia de comercialização. Os produtores priorizam a aparência, ou seja, ausência de defeitos, alta rentabilidade na produção, facilidades de colheita e transporte e resistência a doenças. Os varejistas e atacadistas têm a aparência como atributo mais importante, dando ênfase à firmeza e à boa capacidade de armazenamento. Por sua vez, os consumidores, visam à aparência e às características organolépticas (CHITARRA, M. I. F.; CHITARRA, A. B., 2005).

A qualidade interna do fruto está relacionada com a coloração da polpa, ausência de injúrias e com as características de sabor, aroma e textura, as quais são importantes na sua aceitação final. Sabe-se que esta é resultado das condições climáticas, dos tratos culturais e do estádio de maturação no qual o fruto foi colhido (THÉ et al., 2001).

As características externas de qualidade são importantes na diferenciação do produto, em especial, na decisão de compra. $\mathrm{O}$ sabor, aroma e textura, quando combinados com a aparência, são importantes na aceitação do produto pelo consumidor (CHITARRA; ALVES, 2001).

Estudos sobre a maturação e conservação pós-colheita de frutos são de grande importância, uma vez que estes irão refletir na sua qualidade e no comportamento durante o armazenamento (ABREU; CARVALHO; GONÇALVES, 1998).

A análise sensorial é uma forma rápida e criteriosa de avaliar a qualidade de produtos in natura, pois se baseia na utilização dos

Recebido para publicação em 27/9/2007

Aceito para publicação em 9/3/2008 (002891)

${ }^{1}$ Departamento de Agroindústria, Alimentos e Nutrição, Escola Superior de Agricultura "Luiz de Queiroz", Av. Pádua Dias, 11, CP 9, CEP 13418-900, Piracicaba-SP, Brasil,

E-mail: anaamiguel@yahoo.com.br; ca_abraao@yahoo.com.br

${ }^{*}$ A quem a correspondência deve ser enviada 
órgãos dos sentidos humanos como "instrumentos” de medida para avaliação de atributos físicos e químicos (MEILGAARD; CIVILLE; CARR, 1999).

Assim, neste trabalho, foram avaliadas as alterações nos principais atributos sensoriais de abacaxis 'Pérola' em quatro estádios de maturação durante o armazenamento.

\section{Material e métodos}

Frutos de abacaxi 'Pérola' nos estádios de maturação, "verde", "pintado", "colorido" e "amarelo" (CLASSIFICAÇÃO, 2000) foram adquiridos na CEAGESP (SP), selecionados, levados ao Laboratório de Frutas e Hortaliças da ESALQ/USP e armazenados sob condição ambiente $\left(23,4 \pm 1,7^{\circ} \mathrm{C} ; 70 \%\right.$ UR), por seis dias.

Utilizou-se o delineamento inteiramente casualizado em esquema fatorial $4 \times 4$ (quatro estádios de maturação: verde, pintado, colorido e amarelo; e quatro tempos: 0, 2, 4 e 6 dias de armazenamento), com três repetições, sendo a unidade experimental composta por um fruto. A cada dois dias foram retiradas amostras para análise sensorial da aparência (cor amarela da polpa; defeitos; de maduro; passado), odor (estranho, fermentado; doce), sabor (doce; ácido; de maduro; passado; adstringente; estranho) e textura (firmeza; suculência; presença de fibras na polpa).

Para obter a frequência de consumo e a preferência pelo ponto de consumo, entrevistaram-se 85 clientes do Mercado Municipal de Piracicaba (SP). Os resultados foram anotados como porcentagem em relação ao número total de pessoas consultadas.

A análise sensorial foi feita por uma equipe de 12 provadores treinados, tendo cada um recebido quatro amostras codificadas que correspondiam aos quatro estádios de maturação. Os abacaxis foram retirados do ambiente de armazenamento, descascados, cortados em fatias de aproximadamente $1 \mathrm{~cm}$ de espessura, as quais foram divididas em quatro pedaços e, em seguida, foram acondicionadas em recipientes adequados para prova e servidas aos provadores em cabines individuais. Os resultados das análises individuais de aparência, odor, sabor e textura foram registrados em escala estruturada de nove pontos. As variáveis avaliadas foram submetidas à análise de variância pelo teste $F$ e comparação das médias pelo teste de Tukey a 5\% de probabilidade, de acordo com Gomes (2002).

\section{Resultados e discussão}

O estádio de maturação influenciou significativamente todos os atributos relacionados com a aparência da polpa do abacaxi (Tabela 1). Os frutos no ponto de maturação "amarelo" apresentaram as maiores notas para cor amarela, defeito, maduro e passado. Embora não tenha havido interação significativa entre os estádios de maturação e o tempo de armazenamento nas condições do experimento, os frutos nos estádios "verde" e "pintado" apresentaram polpa de melhor aparência. Isso indica que a qualidade das frutas sofre a influência do estádio de maturação no qual elas são colhidas (KLUGE et al., 2002). Este é um indicativo importante, haja vista que a qualidade da polpa é altamente cotada, tanto no mercado internacional como no interno, podendo proporcionar até $60 \%$ de aumento de preço do produto (USBERTI FILHO, 2005). Como componentes desejáveis citam-se: doçura, acidez de baixa a moderada, consistência tenra, suculência e coloração atraente, ou seja, polpa mais amarela.

A aparência é o atributo que mais causa impacto na escolha por parte do consumidor e, dentro desta, a cor é a característica mais relevante. Isso porque a cor caracteriza sobremaneira o produto, constituindo-se no primeiro critério para sua aceitação ou rejeição. A aparência geral e a cor estão relacionadas com a qualidade, índice de maturação e deterioração do produto (DELIZA, 2000; SANTANA et al., 2004).

Para o odor houve diferença significativa apenas entre os estádios de maturação (Tabela 2). Os resultados mostraram que não foram detectados nos frutos odores estranho e fermentado. Porém, os provadores indicaram que o abacaxi no estádio "amarelo" apresentava aroma doce mais pronunciado, o que era de se esperar, já que, com a maturação, há a evidência do

Tabela 1. Notas médias para atributos de aparência da polpa de frutos de abacaxi 'Pérola', em diferentes estádios de maturação, armazenados à temperatura ambiente $\left(23,4 \pm 1,7^{\circ} \mathrm{C} ; 70 \% \mathrm{UR}\right)$.

\begin{tabular}{lcccc}
\hline \multicolumn{1}{c}{ Variável } & Cor amarela & Defeitos & Maduro & Passado \\
\hline Estádios de maturação & & & & \\
$\quad$ Verde & $5,33^{\mathrm{ab}}$ & $2,75^{\mathrm{b}}$ & $6,00^{\mathrm{b}}$ & $1,63^{\mathrm{b}}$ \\
Pintado & $4,92^{\mathrm{b}}$ & $2,27^{\mathrm{b}}$ & $5,73^{\mathrm{b}}$ & $1,54^{\mathrm{b}}$ \\
Colorido & $5,46^{\mathrm{ab}}$ & $2,81^{\mathrm{b}}$ & $6,06^{\mathrm{b}}$ & $2,25^{\mathrm{ab}}$ \\
Amarelo & $6,13^{\mathrm{a}}$ & $4,33^{\mathrm{a}}$ & $7,23^{\mathrm{a}}$ & $2,83^{\mathrm{a}}$ \\
Dias & & & & \\
0 & $5,81^{\mathrm{a}}$ & $3,38^{\mathrm{a}}$ & $6,48^{\mathrm{a}}$ & $2,29^{\mathrm{a}}$ \\
2 & $5,44^{\mathrm{a}}$ & $3,06^{\mathrm{a}}$ & $5,94^{\mathrm{a}}$ & $2,15^{\mathrm{a}}$ \\
4 & $5,19^{\mathrm{a}}$ & $2,77^{\mathrm{a}}$ & $6,31^{\mathrm{a}}$ & $1,65^{\mathrm{a}}$ \\
6 & $5,40^{\mathrm{a}}$ & $2,96^{\mathrm{a}}$ & $6,29^{\mathrm{a}}$ & $2,17^{\mathrm{a}}$ \\
\hline
\end{tabular}

Cor amarela, maduro, passado ( 1 = nada; 9 = muito); Defeitos ( 1 = nada; 9 = muitos); e médias seguidas de pelo menos uma letra comum, na coluna, não diferem significativamente entre si, pelo teste de Tukey $(\mathrm{p}>0,05)$.

Tabela 2. Notas médias para atributos de odor de frutos de abacaxi 'Pérola', em diferentes estádios de maturação, armazenados à temperatura ambiente $\left(23,4 \pm 1,7^{\circ} \mathrm{C} ; 70 \% \mathrm{UR}\right)$.

\begin{tabular}{cccc}
\hline Variável & Estranho & Fermentado & Doce \\
\hline Estádios de maturação & & & \\
Verde & $1,27^{\mathrm{a}}$ & $1,23^{\mathrm{b}}$ & $5,77^{\mathrm{ab}}$ \\
Pintado & $1,40^{\mathrm{a}}$ & $1,21^{\mathrm{b}}$ & $6,33^{\mathrm{a}}$ \\
Colorido & $1,25^{\mathrm{a}}$ & $1,50^{\mathrm{ab}}$ & $4,90^{\mathrm{b}}$ \\
Amarelo & $1,73^{\mathrm{a}}$ & $2,06^{\mathrm{a}}$ & $6,31^{\mathrm{a}}$ \\
Dias & & & \\
0 & $1,50^{\mathrm{a}}$ & $1,73^{\mathrm{a}}$ & $5,88^{\mathrm{a}}$ \\
2 & $1,40^{\mathrm{a}}$ & $1,40^{\mathrm{a}}$ & $5,38^{\mathrm{a}}$ \\
4 & $1,44^{\mathrm{a}}$ & $1,31^{\mathrm{a}}$ & $6,50^{\mathrm{a}}$ \\
6 & $1,31^{\mathrm{a}}$ & $1,56^{\mathrm{a}}$ & $5,56^{\mathrm{a}}$ \\
\hline
\end{tabular}

Estranho, fermentado ( 1 = nenhum; 9 = muito); doce ( 1 = fraco; 9 = forte); e médias seguidas de pelo menos uma letra comum, na coluna, não diferem significativamente entre si, pelo teste de Tukey $(\mathrm{p}>0,05)$. 
odor característico dos frutos. Analisando-se isoladamente o comportamento desta variável no decorrer do armazenamento, observa-se que o odor se manteve constante durante o período de avaliação, variando de 1,50 a 1,31 ; de 1,73 a 1,56 ; e de 5,88 a 5,56 para as variáveis, estranho, fermentado e doce, respectivamente. De acordo com Awad (1993), com o avanço da maturação, o aroma se acentua devido à síntese de compostos voláteis, sendo indicativo do ponto ideal de consumo.

Em relação ao sabor, houve diferenças significativas para as variáveis maduro, passado e estranho entre os estádios de maturação (Tabela 3). Os frutos no ponto de maturação "amarelo" apresentaram as maiores notas, porém estas não foram suficientes para que os consumidores rejeitassem o produto, indicando que o sabor foi prejudicado pelo estádio avançado de desenvolvimento. De acordo com Thé et al. (2001), a qualidade interna dos frutos e os constituintes físicos e químicos da polpa, responsáveis pelo sabor e aroma característicos, são importantes na sua aceitação final. Chitarra, M. I. F. e Chitarra, A. B. (2005) citam que $\mathrm{o}$ amadurecimento conduz a um aumento na doçura devido ao incremento no teor de açúcares simples, decréscimos da acidez e da adstringência, dada a redução nos conteúdos de ácidos e de compostos fenólicos, respectivamente.

No que se refere à textura, observa-se na Tabela 4 que apenas o atributo fibras foi significativamente afetado pelo grau de maturação, apesar de ter sido verificado aumento nas notas para firmeza e suculência, sendo que o abacaxi "pintado" recebeu as maiores notas para estes parâmetros. Isso indica que o avanço no estádio de maturação não implicou em amaciamento da polpa, uma vez que esta foi compensada pela ausência da perda de suculência. Quanto às fibras, os provadores detectaram maior sensação de fibrosidade nos abacaxis "amarelos", o que pode ser indicativo de senescência.

A análise sensorial também permitiu verificar que a textura dos abacaxis, em diferentes estádios de maturação, não diferiu significativamente durante o período de armazenamento. Segundo Chitarra, M. I. F. e Chitarra, A. B. (2005), a textura é um dos atributos de qualidade mais importantes, pois está relacionada ao sabor.
Constatou-se que $42,9 \%$ dos entrevistados consomem abacaxi uma vez por semana, $8,6 \%$ duas vezes, $11,4 \%$ a cada quinze dias, $28,6 \%$ uma vez por mês, $2,9 \%$ a cada dois meses e 5,7\% a cada três meses. Tal informação é importante tanto aos varejos (tipo quitanda e sacolões) quanto às redes de supermercado, pois podem constituir uma logística de venda que atenda à demanda e satisfaça os consumidores.

Sempre que possível, as frutas e hortaliças devem ser submetidas ao teste de preferência para se conhecer a reação dos consumidores e satisfazer suas exigências. Quando questionados sobre o abacaxi que desejam adquirir, identificouse que o consumidor associa a coloração da casca com o grau de maturação da fruta, pois $60,0 \%$ dos entrevistados preferem abacaxis de casca verde-amarelada a amarelada, concordando com o indicado por Chitarra, M. I. F. e Chitarra, A. B. (2005), que os produtos de cor forte e brilhante são os mais procurados pelos consumidores, pois estes correlacionam às mudanças de coloração, por ocasião do amadurecimento, com o aumento da doçura e das características sensoriais. Dessa maneira, normalmente a decisão de compra recai sobre produtos

Tabela 4. Notas médias para atributos da textura de frutos de abacaxi 'Pérola', em diferentes estádios de maturação, armazenados à temperatura ambiente $\left(23,4 \pm 1,7^{\circ} \mathrm{C} ; 70 \% \mathrm{UR}\right)$.

\begin{tabular}{lccc}
\hline \multicolumn{1}{c}{ Variável } & Firmeza & Suculência & Fibras \\
\hline Estádios de maturação & & & \\
Verde & $6,58^{\mathrm{a}}$ & $6,92^{\mathrm{a}}$ & $5,66^{\mathrm{ab}}$ \\
Pintado & $7,10^{\mathrm{a}}$ & $7,44^{\mathrm{a}}$ & $4,54^{\mathrm{b}}$ \\
Colorido & $6,69^{\mathrm{a}}$ & $6,94^{\mathrm{a}}$ & $5,88^{\mathrm{ab}}$ \\
Amarelo & $6,33^{\mathrm{a}}$ & $7,31^{\mathrm{a}}$ & $6,12^{\mathrm{a}}$ \\
Dias & & & \\
0 & $6,35^{\mathrm{a}}$ & $7,29^{\mathrm{a}}$ & $5,92^{\mathrm{a}}$ \\
2 & $6,52^{\mathrm{a}}$ & $6,77^{\mathrm{a}}$ & $5,33^{\mathrm{a}}$ \\
4 & $6,77^{\mathrm{a}}$ & $7,15^{\mathrm{a}}$ & $4,92^{\mathrm{a}}$ \\
6 & $7,06^{\mathrm{a}}$ & $7,40^{\mathrm{a}}$ & $6,04^{\mathrm{a}}$ \\
\hline
\end{tabular}

Firmeza ( $1=$ pouco; $9=$ muito); suculência $(1=$ pouca; $9=$ muita $)$; presença de fibras ( 1 = nenhuma; 9 = muitas); e médias seguidas de pelo menos uma letra comum, na coluna, não diferem significativamente entre si, pelo teste de Tukey $(p>0,05)$.

Tabela 3. Notas médias para atributos do sabor de frutos de abacaxi 'Pérola', em diferentes estádios de maturação, armazenados à temperatura ambiente $\left(23,4 \pm 1,7^{\circ} \mathrm{C} ; 70 \% \mathrm{UR}\right)$.

\begin{tabular}{|c|c|c|c|c|c|c|}
\hline Variável & Doce & Ácido & Maduro & Passado & Adstringente & Estranho \\
\hline \multicolumn{7}{|c|}{ Estádios de maturação } \\
\hline Verde & $5,69^{\mathrm{a}}$ & $3,94^{\mathrm{a}}$ & $5,94^{\mathrm{b}}$ & $1,29^{c}$ & $2,27^{\mathrm{a}}$ & $1,13^{\mathrm{b}}$ \\
\hline Pintado & $6,06^{\mathrm{a}}$ & $4,33^{\mathrm{a}}$ & $6,66^{\mathrm{ab}}$ & $1,48^{\mathrm{bc}}$ & $2,31^{\mathrm{a}}$ & $1,15^{\mathrm{ab}}$ \\
\hline Colorido & $5,50^{\mathrm{a}}$ & $3,96^{\mathrm{a}}$ & $6,19^{\mathrm{b}}$ & $2,18^{\mathrm{ab}}$ & $2,19^{\mathrm{a}}$ & $1,21^{\mathrm{ab}}$ \\
\hline Amarelo & $6,27^{\mathrm{a}}$ & $4,13^{\mathrm{a}}$ & $7,31^{\mathrm{a}}$ & $2,31^{\mathrm{a}}$ & $2,35^{\mathrm{a}}$ & $1,50^{\mathrm{a}}$ \\
\hline \multicolumn{7}{|l|}{ Dias } \\
\hline 0 & $6,08^{\mathrm{a}}$ & $4,02^{\mathrm{a}}$ & $6,65^{\mathrm{a}}$ & $2,02^{\mathrm{a}}$ & $2,42^{\mathrm{a}}$ & $1,31^{\mathrm{a}}$ \\
\hline 2 & $5,66^{\mathrm{a}}$ & $3,81^{\mathrm{a}}$ & $6,40^{\mathrm{a}}$ & $1,88^{\mathrm{a}}$ & $2,50^{\mathrm{a}}$ & $1,21^{\mathrm{a}}$ \\
\hline 4 & $6,06^{\mathrm{a}}$ & $3,81^{\mathrm{a}}$ & $6,42^{\mathrm{a}}$ & $1,44^{\mathrm{a}}$ & $1,98^{\mathrm{a}}$ & $1,13^{\mathrm{a}}$ \\
\hline 6 & $5,71^{\mathrm{a}}$ & $4,71^{\mathrm{a}}$ & $6,65^{\mathrm{a}}$ & $1,94^{\mathrm{a}}$ & $2,23^{\mathrm{a}}$ & $1,33^{\mathrm{a}}$ \\
\hline
\end{tabular}

Doce, ácido, adstringente ( 1 = pouco; 9 = muito); maduro, passado ( 1 = nada; $9=$ muito); estranho ( 1 = nenhum; $9=$ muito); e médias seguidas de pelo menos uma letra comum, na coluna, não diferem significativamente entre si, pelo teste de Tukey $(\mathrm{p}>0,05)$. 
coloridos, no caso do abacaxi, principalmente sobre aqueles que apresentam a coloração da casca tendendo ao amarelo.

A aparência foi uma das características mais indicadas pelos consumidores, $14,3 \%$ dos entrevistados, o que vem ao encontro do enunciado por Kader (2002): que 83\% da decisão de adquirir ou rejeitar um produto é determinada pela aparência ou condição em que se encontra.

A prática de apertar o abacaxi para verificar sua integridade é realizada por $20,0 \%$ dos entrevistados; $8,6 \%$ observam e recusam abacaxis passados; $17,1 \%$ observam se há defeitos na casca do abacaxi, incluindo danos mecânicos (perfurações, amassados) ou qualquer anormalidade externa evidente. Também se constatou que o tamanho é observado por $17,1 \%$ dos compradores de abacaxi.

\section{Conclusões}

O estádio de maturação afetou a qualidade sensorial do abacaxi. Tanto a aparência da polpa quanto a qualidade sensorial foram afetadas negativamente pelo estádio de maturação, atingindo maior grau de qualidade sensorial nos pontos "pintado" e "colorido".

\section{Referências bibliográficas}

ABREU, C. M. O.; CARVALHO, V. D.; GONÇALVES, N. B. Cuidados pós-colheita e qualidade do abacaxi para exportação. Informe Agropecuário, v. 19, n. 195, p. 70-72, 1998.

AGRIANUAL: Anuário da agricultura brasileira. In: Abacaxi. São Paulo: FNP, Consultórios e Comércio, 2007. p. 149-158.

AWAD, M. Fisiologia Pós-colheita de frutos. São Paulo: Nobel, 1993.

CHITARRA, A. B.; ALVES, R. E. Tecnologia de pós-colheita para frutas tropicais. Fortaleza: Frutal - Sindifruta, 2001.

CHITARrA, M. I. F.; CHITARRA, A. B. Pós-colheita de frutas e hortaliças: fisiologia e manuseio. 2 ed. Lavras: UFLA, 2005.
CLASSIFICAÇÃO do abacaxi (Ananas comosus (L.) Merril). Recife: Centro de qualidade em Horticultura, CEAGESP, 2000. (Folder).

DELIZA, R. Importância da qualidade sensorial em produtos minimamente processados. In: ENCONTRO NACIONAL SOBRE PROCESSAMENTO MÍNIMO DE FRUTAS E HORTALIÇAS, 2, 2000. Palestras... Viçosa: UFV, 2000. p. 73-74.

USBERTI FILHO, J. A. U. Novo abacaxi. In: Instituto Agronômico De Campinas - IAC. Disponível em:<http:www.iac.gov.br/Arquivos/ Noticias/Materias/NovoAbacaxi.htm>. Acesso em: 22 fevereiro 2005.

GIACOMINO, A. Comunicação pessoal (10 outubro 2001).

GOMES, F. P. Estatística aplicada a experimentos agronômicos e florestais. Piracicaba: FEALQ, 2002.

KADER, A. A. Postharvest technology of horticultural crops. 3 ed. Davis: University of California, 2002.

KLUGE, R. A. et al. Fisiologia e manejo pós-colheita de frutas de clima temperado. Pelotas: UFPEL, 2002.

MEILGAARD, M.; CIVILLE, G. V.; CARR, B. T. Sensory Evaluation Techniques. 3 ed. Boca Raton: CRC Press, 1999.

REINHARDT, D. H.; SOUZA, L. F. S.; CUNHA, G. A. P. Exigências edafoclimáticas. In: Frutas do Brasil 7. Abacaxi: produção. Cruz das Almas: Empresa Brasileira de Pesquisa Agropecuária, Embrapa Mandioca e Fruticultura, 2000. Cap. 3, p.11-12.

SANTANA, L. L. A. et al. Efeitos de modo de aplicação e concentrações de etefon na coloração da casca e outros atributos de qualidade do abacaxi 'Pérola'. Revista Brasileira de Fruticultura, v. 26, n. 2, p. 212-216, 2004.

SOUTO, R. F. et al. Conservação pós-colheita do abacaxi 'Pérola' colhido no estádio de maturação "pintado" associando-se refrigeração e atmosfera controlada. Revista Brasileira de Fruticultura, v. 26, n. 1, p. 24-28, 2004.

THÉ, P. M. P. et al. Efeito da temperatura de armazenamento sobre a composição química do abacaxi cv. Smooth Cayenne L. Ciência e Agrotecnologia, v. 25, n. 2, p. 356-363, 2001. 